\title{
Le Journal d'un émigré : Robert de Saint-Vincent entre jansénisme et Contre-Révolution
}

The Journal of an Emigre: Robert de Saint-Vincent between Jansenism and Counterrevolution

\section{Valérie Guittienne-Mürger et Monique Cottret}

\section{(2) OpenEdition}

\section{Journals}

\section{Édition électronique}

URL : https://journals.openedition.org/ahrf/12865

DOI : 10.4000/ahrf.12865

ISSN : 1952-403X

Éditeur :

Armand Colin, Société des études robespierristes

Édition imprimée

Date de publication : 1 septembre 2013

Pagination : 101-122

ISBN : 9782200928261

ISSN : 0003-4436

\section{Référence électronique}

Valérie Guittienne-Mürger et Monique Cottret, «Le Journal d'un émigré : Robert de Saint-Vincent entre jansénisme et Contre-Révolution », Annales historiques de la Révolution française [En ligne], 373 । juilletseptembre 2013, mis en ligne le 01 septembre 2016, consulté le 01 juillet 2021. URL : http://

journals.openedition.org/ahrf/12865 ; DOI : https://doi.org/10.4000/ahrf.12865 


\title{
LE JOURNAL D'UN ÉMIGRÉ : ROBERT DE SAINT-VINCENT ENTRE JANSÉNISME ET CONTRE-RÉVOLUTION
}

Valérie GUITTIENNE-MÜRGER et Monique COTTRET

\begin{abstract}
Qui était Robert de Saint-Vincent (1725-1799) ? L'historiographie présente deux images différentes du personnage, d'une part le bouillant opposant à la monarchie absolue, le sévère janséniste qui défend la tolérance civile à l'égard des protestants, et d'autre part l'homme du 25 septembre 1788, celui qui veut fondre 1789 dans le moule de 1614. Les ego-documents redécouverts récemment (ses Mémoires rédigés en émigration et un fragment de correspondance pour les années 1789-1790) permettent de retrouver la cohérence de l'individu et de son système. Robert de Saint-Vincent croit en la monarchie de justice et interprète comme despotisme ministériel toute volonté absolutiste. Pas de roi sans Parlement. Dès lors, on comprend son désarroi, l'impression d'être trahi par les siens et la perception de la Révolution comme le mal absolu, un immense complot jésuite.
\end{abstract}

Mots-clés : Parlement, magistrat, contre-révolution, émigration, jansénisme, jésuites.

Le magistrat janséniste Pierre Augustin Robert de Saint-Vincent (1725-1799) est présenté de façon particulièrement acide dans le Dictionnaire du temps des Lumières : «[...] personnage médiocre et acrimonieux, il se pose sous le règne de Louis XVI en défenseur de la tradition anti- despotique et janséniste du Parlement [...]. Courageux face à la monarchie déclinante, il le sera moins devant la Révolution : dès 1789, 
il s'empressera d'émigrer... »'. Une esquisse psychologique (médiocre, acrimonieux, poseur, lâche) est supposée rendre compte des aléas des engagements du vieux militant parlementaire. Dans son cas, en effet, l'anti despotisme ne conduit pas à la Révolution, mais à l'émigration, sinon en 1789 , du moins relativement tôt en 1790 . Homme de contradiction? Apprenti sorcier sans envergure ? Les choses ne sont pas si simples; il est difficile de cerner précisément l'action de Robert de Saint-Vincent sous le règne de Louis XVI. Il demeure certes aux premières loges de l'opposition parlementaire. On connaît son plaidoyer en faveur de l'octroi d'un état civil aux protestants ${ }^{2}$, il se prononce même pour le retour à l'édit de Nantes, ce qui allait plus loin que la volonté royale en autorisant la liberté de culte que ne comportait pas l'édit de 1787. Mais en-dehors de cette intervention particulière, ses prises de position ont été rapportées par des témoignages extérieurs et souvent tardifs.

Les Mémoires de Robert de Saint-Vincent que nous avons retrouvés et publiés fournissent de précieux renseignements sur le règne de Louis $\mathrm{XV}$, notamment sur les liens de Choiseul et de la mouvance janséniste. Ils demeurent très allusifs quant à son action sous le dernier règne de la monarchie absolue ${ }^{3}$. Ayant récemment pu utiliser une partie de la correspondance de Robert de Saint-Vincent pour les années 1789-1790, nous ajoutons cet ensemble au dossier pour mieux comprendre l'homme face à l'événement. L'écriture immédiate rejoint celle des mémoires. Une cohérence se dégage, celle d'un vieil homme aux prises avec une jeunesse qu'il ne comprend plus dans un monde dont les valeurs nouvelles lui sont totalement étrangères ${ }^{4}$.

(1) Jean de VIGUERIE, Histoire et Dictionnaire du temps des Lumières, 1715-1789, Paris, Bouquins, Robert Laffont, 1995, p. 1335.

(2) Monique COTTRET, « La tolérance, une fleur du sectarisme ? », Homo religiosus, autour de Jean Delumeau, Paris, Fayard, 1997, p. 460-467.

(3) Monique CotTret, Valérie GuITIENNE-Mürger, Nicolas LyON-CAEN, Un magistrat janséniste du siècle des Lumières à l'Émigration, Pierre-Augustin Robert de Saint-Vincent, Bordeaux, Presses universitaires de Bordeaux, 2012. L'ouvrage comprend le Mémoire de famille (désormais I) et le Mémoire pour sa femme (désormais II).

(4) Nous remercions Muriel Van Kempen de nous avoir communiqué cette correspondance. Voir Muriel VAN KEMPEN, «Les exilés de l'Unigenitus et l'Église de Hollande (1725-1745) », thèse de doctorat sous la direction de Monique COTTRET, Université Paris-Ouest-Nanterre, 2011 ; le correspondant de Robert de Saint-Vincent est l'abbé Clément, voir à ce sujet la thèse de l'école des Chartes d'Emmanuel LACAM, sous la direction de Monique COTTRET et Olivier PONCET, « Entre Rome et Madrid un réformateur janséniste dans l'Europe méditerranéenne. Correspondances et voyages d'un intermédiaire culturel l'abbé Jean-Charles Augustin Clément (1754-1771) », 2013. 


\section{Comment Robert de Saint-Vincent devient contre-révolutionnaire}

Pour Robert de Saint-Vincent, tout bascule en septembre 1788, précisément le 25 septembre. En août Necker a été appelé aux Finances, le 14 septembre Lamoignon doit démissionner, le 23 septembre une Déclaration royale abandonne la réforme judiciaire dite de Lamoignon et rappelle que les États généraux sont convoqués pour le $1^{\mathrm{er}}$ mai 1789. De retour d'exil, le Parlement fait sa rentrée le 24 septembre. Le 25, il perd une grande partie de sa popularité en demandant que les États généraux soient réunis «en suivant la forme observée en 1614 » et ceci sur proposition de Robert de Saint-Vincent en dépit des protestations de Duport ${ }^{5}$. C'est la dernière fois que Robert emporte une décision et cela sera sans conséquence puisque le 27 décembre 1788, c'est le Conseil du roi qui se prononce en faveur du doublement de la représentation du Tiers aux États généraux et donc choisit l'innovation contre la tradition. Robert de Saint-Vincent se retrouve sur la position des princes du sang qui dès le 12 décembre avaient remis au roi un mémoire qui défendait le vote par ordre et la délibération séparée, et donc anéantissait les effets virtuels du doublement du Tiers.

Pourtant les historiens considèrent généralement que Robert de Saint-Vincent, en dépit de cette déclaration, aurait rejoint le club qui se réunissait chez le conseiller Duport et que l'on appelle parfois la «société des Trente $»^{6}$. Si tel est bien le cas, Robert de Saint-Vincent assista sans doute aux premières réunions en novembre 1788 , mais abandonna vite ; le 4 décembre 1788, Mirabeau souhaite d'ailleurs mettre cette société à l'abri de « la tyrannie parlementaire $»^{7}$. Et dans une lettre du 21 septembre 1789 , Robert dénonce cette entreprise : « Voilà ce que nous avons vu se former, se cimenter, se fortifier cet hiver depuis l'idée des États généraux chez M. Duport, conseiller au Parlement, rue du Grand Chantier au marais. C'est là où se sont réunis Target, La Fayette, Mathieu de Montmorency, La Rochefoucauld le duc, le vicomte de Noailles, et autres très connus pour avoir conçu le projet de détruire, comme le dit Target, huit siècles de préjugés en huit séances d'États généraux. Remarquons, je vous prie,

(5) Jean EGRET, La Pré-Révolution française, 1787-1788, Paris, PUF, 1962, p. 338.

(6) Les historiens suivent en cela les témoignages de Montmorency-Luxembourg, Barentin, Morellet, La Fayette, voir Jean EGRET, op. cit., p. 327. Yann Fauchois propose la liste suivante pour les principaux membres du club constitutionnel qui se réunit chez Adrien Duport le 10 novembre 1788 : Condorcet, Dupont de Nemours, Target, Lacretelle, Roederer, Duval d'Epremesnil, Robert de Saint-Vincent, La Fayette, Mirabeau, Talleyrand, Noailles, Aiguillon, Lauzun de Biron, Castellane, Latour-Maubour, Destutt de Tracy, Beauvau, La Rochefoucauld, Montmorency-Luxembourg, Yann FAUCHOIS, Chronologie politique de la Révolution, Paris, Marabout université, 1989, p. 25.

(7) Jean EGRET, op. cit., p. 328-329. 
que la plupart de tous ces convives n'ont d'autre religion que la religion nouvelle, d'autre catéchisme que celui de Jean-Jacques, d'autre projet que de regarder la France comme un terrain tout neuf $[. ..] \gg^{8}$. Robert de Saint-Vincent l'a emporté le 25 septembre 1788 contre Adrien Duport mais en-dehors du Parlement c'est désormais ce dernier qui donne le ton. La jeunesse parlementaire rejoint la noblesse libérale et tous se mettent au service du Tiers contre les ordres privilégiés. Si Robert de Saint-Vincent a bien été parmi les « convives » réunis par Duport, les échanges ont dû être vifs ! Entre le vieux magistrat janséniste et le jeune Duport, mesmérien et franc-maçon, la distance était trop grande ${ }^{9}$. Il est également particulièrement hostile à Target ${ }^{10}$, avocat au parlement de Paris depuis 1752, avec lequel il a partagé le combat contre Maupeou, mais qui a adopté le style démagogue, et renoncé à l'érudition pour défendre la politique de la table rase : une véritable trahison dans l'esprit de Robert qui poursuit en déplorant la « confusion des ordres », la fin des privilèges et la violence des institutions nouvelles ${ }^{11}$. Robert de Saint-Vincent demeure du côté du mandat impératif, se méfie de la populace et ne supporte pas la publicité des débats. Il a déjà choisi son camp. Mais ce qui le choque plus encore, c'est la politique religieuse de l'assemblée : "Voilà la vraie source de la faiblesse et de la complaisance du clergé qui le 4 août a consenti à tout parce que chaque prélat était menacé de périr dans trois jours s'il ne consentait à la suppression des dîmes et à la destruction de toute propriété ecclésiastique qu'on dit aujourd'hui être du domaine de la nation. La faiblesse et l'ignorance du clergé ont grandement secondé la crainte qui leur a été inoculée [...] $\gg^{12}$. Et comment attendre mieux du clergé formaté par les tenants de la bulle Unigenitus ? Pourtant le plus douloureux est encore à venir ! Robert est navré par ce qu'il considère comme la trahison des « appelants ». Ceux qui depuis l'appel de 1717 à un concile général luttent contre la Bulle et les jésuites, ses amis, les amis de la vérité, les disciples de saint Augustin, se retrouvent dans le mouvement révolutionnaire, dans la sédition pour reprendre son vocabulaire. C'est un cri de détresse que Robert de SaintVincent lance à son correspondant, l'abbé Clément, pour réaliser que

(8) Lettre 20, 21 septembre 1789, Archives d'Utrecht, Fonds Port-Royal (ancien fonds d'Amersfoort), PR 1726 (Amf. 5). La cote recouvre un ensemble de 32 lettres. Le texte a été modernisé.

(9) Marcel DorignY, « Duport, Adrien, Jean-François, 1759-1798 », dans Albert SoBOUL (dir.), Dictionnaire historique de la Révolution française, Paris, PUF, 1989, p. 382-383.

(10) Guy Jean-Baptiste Target (1733-1806) est l'une des gloires du barreau et membre de l'Académie depuis 1780 ; il a également été l'avocat du cardinal de Rohan « le cardinal collier », et à cette occasion s'est à nouveau retrouvé en accord avec Robert.

(11) Lettre 20, op. cit.

(12) Ibidem. 
lui-même, ce « cher abbé », s'inscrit dans les principes nouveaux. Du coup, Robert va les désigner par le terme de « jansénistes », expression que les jansénistes récusent en temps normal car elle les transforme en sectateurs de Jansenius, alors qu'ils se considèrent comme les seuls vrais catholiques : « Mais dites-moi donc quelle est la fureur de nos jansénistes de se livrer à corps perdu dans la fermentation que le ministère excite dans le Tiers de tous côtés pour manger les gros par les petits et les croquer tous ? $\gg^{13}$. Devant cet abandon, Robert de Saint-Vincent interprète la situation comme un complot universel :

« Nous n'aurions jamais cru voir les appelants de nos jours d'accord avec les jésuites, les protestants, les philosophes, les académies, les Cerutti, les Morellet, les Maury. Le Parlement était adoré il y a six mois, c'est aujourd'hui à qui des jésuites ou des jansénistes lui jettera la première pierre. On veut le détruire. Son anéantissement est regardé comme un bien public. Tout le monde veut en avoir l'honneur $»^{14}$.

Robert ne peut accepter les principes nouveaux, et il souffre particulièrement de voir les jeunes conseillers des Enquêtes abandonner la cause du Parlement pour celle de la nation, de constater que les appelants rejoignent massivement la Révolution ${ }^{15}$. Dans son esprit cependant, il ne s'agit pas d'un reniement de ses combats antérieurs ; il a toujours lutté contre le despotisme ministériel, au nom du roi de justice, au nom du roi des juges ${ }^{16}$; lorsqu'il décrit dans ses Mémoires la fameuse séance de la flagellation, au cours de laquelle Louis XV vient réaffirmer son pouvoir absolu devant le Parlement, il voit un roi honteux du rôle qu'on lui fait tenir ${ }^{17}$... Pour Robert de Saint-Vincent c'est du pur despotisme ! Robert interprète donc à nouveau la politique de Necker selon les mêmes principes et s'y oppose toujours au nom du droit mais cette fois il est surpris de ne plus trouver l'opinion derrière le Parlement :

(13) Lettre 3, 4 janvier 1789.

(14) Ibidem.

(15) Nous avons estimé que les appelants se scindent une nouvelle fois devant le mouvement révolutionnaire, et que deux-tiers des jansénistes rejoignent le Tiers et suivent la politique de l'Assemblée. Robert de Saint-Vincent semble penser que la quasi-totalité des jansénistes a choisi le camp de la Révolution, mais évidemment les positions vont changer à partir du moment où se construit l'Église constitutionnelle. Monique COTTRET, «1789-1791 : triomphe ou échec de la minorité janséniste ? », Christine PEYRARD (textes réunis par), Révolution et minorité religieuse, Rives nord-méditerranéennes, n¹4, Aix-en-Provence, Telemme, 2003, p. 49-61.

(16) Michel FIGEAC, «Les magistrats en révolte en 1789 ou la fin du rêve politique de la monarchie des juges », Histoire, économie et société, 2006, $\mathrm{N}^{\circ}$ 25-3, p. 385-400.

(17) I, p. 407-408. 
«Bref on veut détruire plus sûrement que Richelieu et Mazarin l'influence du clergé et de la noblesse, et se servir de la patte des petits du Tiers état pour les croquer tous les uns après les autres, et les assujettir au despotisme [...]. Et voyez ceux qui prêchent cette nouvelle doctrine, ce sont tous les jésuites. Il n'y en a pas un seul connu dans la société qui ne soit de cet avis. Cerruti ${ }^{18}$ et ses semblables en sont les prédicateurs. Les protestants qui ne sont pas apôtres de l'autorité royale s'animent dans tous les lieux où ils ont du pouvoir. Ils espèrent plus du peuple Tiers état que du clergé et de la noblesse le rétablissement du culte public qu'ils sollicitent avec vivacité. Ils l'obtiendront plus facilement d'une seule assemblée que des trois ordres séparés dont un seul aura le veto. Les philosophes sont ou jésuites ou protestants. Toutes les académies incrédules sont Tiers état par principe. Voilà ceux que M. Necker favorise en établissant que le vœu du Tiers sera toujours le vœu général et national. À regarder les choses de près convenez qu'il faut se mettre au-dessus des idées populaires» ${ }^{19}$.

Richelieu, Mazarin, Necker, trois figures de la construction du despotisme selon Robert de Saint-Vincent et de la tentative d'alliance du trône, ou du moins du ministère, avec les « petits » contre les privilégiés. Robert est bien dans la tradition des élites et du devoir de révolte analysé par Arlette Jouanna ${ }^{20}$. Il semble même se figer un peu dans la résistance : en 1787 lui-même envisageait le rétablissement du culte protestant, certes dans un contexte moins agité. Mais surtout Robert de Saint-Vincent attend une « contre révolution ${ }^{21}$, c'est-à-dire une reprise en main brutale par le pouvoir ministériel, un super coup Maupeou-Lamoignon. Dans son esprit l'alliance du Tiers et du trône est un leurre et le ministère attend son heure, celle de la revanche et de la répression :

« Mais enfin, pourquoi cette assemblée dont le Parlement avait conçu de si grandes espérances, dont toutes les provinces du royaume espéraient

(18) Joseph Antoine Joachim Cerutti (1738-1792) est l'un des très rares ex-jésuites à avoir rejoint le camp de la Révolution. On estime qu'il a rompu avec la société autour de 1767. Contrairement à ce qu'affirme Robert de Saint-Vincent l'immense majorité des jésuites se retrouvent dans l'entourage de Pie VI et sont très hostiles à la Révolution française, Gérard PELLETIER, Rome et la Révolution française. La théologie et la politique du Saint-Siège devant la Révolution française (1789-1799), Rome, École française de Rome, $n^{\circ} 319$, 2004. Monique COTTRET, « Les droits de l'homme en enfer », dans Monique CotTret, Caroline Galland, Les damnés du ciel et de la terre, Limoges, PULIM, 2010, p. 219-228.

(19) Lettre 3, op. cit.

(20) Arlette JounnNa, Le devoir de révolte, la noblesse française et la gestation de l'État, Paris, Fayard, 1989.

(21) Lettre 20, op. cit. 
leur salut, dont tout bon citoyen a désiré la réunion pour espérer le salut public auquel on ne voyait aucun autre espoir, pourquoi ne réussit-elle pas ? Pourquoi en est-on à désirer sa séparation, sa dissolution, sa liquéfaction. Il est difficile de ne pas croire que le gouvernement ait eu envie de la dissoudre au mois de juillet dernier. Ce projet seul mal imaginé, encore plus mal projeté, a opéré une révolution sous laquelle l'autorité royale a plié et est comme ensevelie ; qu'avons-nous à craindre de la contre révolution qu'il est impossible de ne pas prévoir. C'est ce que chacun pense ici tous les jours en imaginant tous les matins qu'on est heureux d'avoir prolongé jusque-là son existence $»^{22}$.

Robert de Saint-Vincent n'est ni révolutionnaire, ni contrerévolutionnaire, dans son esprit du moins. Il est fidèle à la monarchie traditionnelle et prêt à disparaitre avec elle, en novembre 1789 il écrit : « je louerai fort le Parlement de périr en même temps que l'autorité et la liberté personnelle du roi. C'est le plus superbe de tous les épitaphes $»^{23}$. En attendant il semble désireux de se retirer des affaires et de vivre le plus possible loin de Paris.

\section{Entre Paris et Montargis}

Robert de Saint-Vincent a quitté Paris en septembre 1789, espérant trouver dans sa terre de Fessard un refuge tranquille. Vain espoir ! L'effervescence règne partout. Les accusations de «monopoleur de blé » portées contre lui depuis vingt ans dans la province de Montargis reprennent et le désignent comme la « cause de la misère du peuple et de la cherté du pain $»^{24}$. Les premières attaques contre ses privilèges ne tardent pas : on coupe les arbres qu'il plante, on brise les cadenas du réservoir à poisson, « ce qui n'aurait jamais été fait sans la Révolution » ${ }^{25}$. Il constate vite « que la conjuration était formée ». Le plus douloureux est sans doute de découvrir que ce sont ses « obligés » qui mènent la danse : «J'ai été, comme mes voisins et comme les seigneurs des différentes provinces du royaume, victime de la fureur de ceux que j'avais le plus obligés et qui m'auraient été le plus fidèles, si la fureur de la Convention (sic, il confond les temps et les assemblées, en l'occurrence il s'agit de la Constituante) n'avait pas 
tout à fait renversé leurs têtes ${ }^{26}$. » Sont particulièrement acharnés, selon lui : le curé27 qui lui doit sa cure, le syndic Georges Drouin, le notaire François-Marie Charrier de Conchard, le fils de sa cuisinière qu'il a fait élever et qui, dans l'assemblée de district de Nemours, a " appris tout ce qu'il fallait faire pour faire de la peine aux seigneurs et les réduire à être dans les principes de l'égalité comme le dernier des habitants $»^{28}$. Durant l'année 1790, Robert, vieux routier de la «persécution », fait profil bas et ne demande qu'à s'enfermer dans son château ${ }^{29}$. Mais il reste une figure importante et ne peut se soustraire à certaines obligations, comme d'assister à l'assemblée populaire pour l'élection de la municipalité ${ }^{30}$, puis à la première assemblée municipale ${ }^{31}$.

Robert de Saint-Vincent se dit dégoûté de cette terre qu'il a pourtant du mal à abandonner ${ }^{32}$. Pour échapper à cette atmosphère détestable, sa femme et lui décident début avril 1790 de faire la tournée de toutes les fermes qu'ils possèdent l'un ou l'autre en pays chartrain et dans l'Orléanais. À l'évidence, ils n'entendent pas renoncer à ce qu'ils considèrent encore comme leurs droits. Chemin faisant, ils rendent visite à leurs amis et mesurent alors le fossé qui se creuse entre eux et les partisans de la Révolution, comme les Laideguive «enfarinés de la nouvelle doctrine démocratique $»^{33}$. Leur vie sociale se complique donc singulièrement et s'ils veillent tous deux à éviter « toute espèce de discussion et de dissertation sur toutes ces matières $»^{34}$, leur réticence même les signale comme des adversaires de la nouveauté.

Résistance passive ou activisme ? Dans le cas de Robert, si discret qu'il se fasse dans sa province, son action à Paris est bien celle d'un parlementaire contre-révolutionnaire. L'historiographie ne mentionne généralement pour Paris, dans la série des protestations des parlements en 1789-1790 $0^{35}$, que la déclaration secrète de la Chambre des Vacations du 14 octobre 1790. Robert de Saint-Vincent nous apprend que les magistrats de la Grand'Chambre ont rédigé une protestation secrète en date du 6

(26) Ibidem.

(27) II, p. 180.

(28) II, p. 204

(29) II, p. 200

(30) II, p. 202

(31) II, p. 207.

(32) II, p. 180.

(33) II, p. 185.

(34) Ibidem.

(35) Sur les différentes protestations et les condamnations qu'elles ont entrainé, voir HenriBruno BASTARD-D'ESTANG, Les parlements de France. Essai historique sur leurs usages, leur organisation et leur autorité, Paris, Didier \& Cie, 1858, t. 2, p. 642-704. 
avril 1790 qui semble n'avoir jamais été découverte. Cette protestation « conjointe et uniforme contre toutes les entreprises de l'Assemblée nationale et la destruction prochaine de la royauté à laquelle il était impossible de ne pas voir qu'on cheminait à grands pas $»^{36}$, dont Robert et d'Esprémesnil auraient été les principaux rédacteurs, fut confiée à notre auteur. C'est chez lui que les magistrats « avertis prudemment » vinrent la lire et la signer. D'après Robert de Saint-Vincent, à son départ de Paris en avril, il y avait entre 60 et 70 signatures et seuls Clément de Blavet et M. Desponty de Saint-Avoye s'étaient abstenus. Laverdy lui-même aurait donné pouvoir à un ami pour signer ${ }^{37}$. Quelques magistrats de la Chambre des Vacations furent invités à les rejoindre, mais nous dit Robert : « ils s'en défendirent tous sous différents prétextes. Je leur dois justice, ce n'était point dissemblance d'opinions, mais il y avait entre eux une convention de faire quelque démarche entre eux au moment de leur dissolution $»^{38}$ Confiée à deux conseillers originaires de Saint-Domingue récemment reçus au Parlement, MM. Merceron et Rossignol, cette mystérieuse protestation aurait été remise à Monsieur et au comte d'Artois à Coblence ${ }^{39}$.

Plus prévoyants que beaucoup d'autres, sans doute formés par les divers exils imposés à Robert depuis son entrée au Parlement, les SaintVincent profitent d'un séjour parisien pour « arranger leurs affaires ». Ils fuient Paris pour éviter la fête de la Fédération, même cet épisode de réconciliation et d'unanimité célébrée leur est étranger. Ils sont presque contraints d'y participer à Évry-les-Châteaux ${ }^{40}$ et finissent par retourner à Fessard où la situation se dégrade encore. Bientôt, on leur conseille de ne plus se promener dans leur bois : un coup de fusil est si vite arrivé. Puis, il leur faut garder leurs contrevents fermés pour ne pas être une cible trop tentante ${ }^{41}$. Mme de Saint-Vincent, quant à elle, profite « de ce temps qu'elle regardait pour elle comme un temps de tranquillité et de calme pour engager plusieurs de ses habitants qui étaient en retard de payer les cens et rentes et les droits seigneuriaux [...] malgré tout ce qu'on disait à

(36) II, p. 181.

(37) II, p. 183.

(38) Ibidem. Bien informé du fond de sa retraite, il rapporte ce qu'il est advenu des signataires de la protestation de la Chambre des Vacations, conservée par Louis Le Peletier de Rosanbo, découverte chez lui sur dénonciation d'un valet en frimaire an II, qui fut à l'origine du procès des magistrats en floréal suivant (AN, Série W 349 et 527). Robert attribue à Robespierre la volonté de les « exterminer jusqu'au dernier », II, p. 184.

(39) Ibidem.

(40) I.e. Évry Grégy-sur-Yerre, II, p. 193-197.

(41) II, p. 214. 
Montargis et dans les paroisses voisines contre les seigneurs et les droits seigneuriaux $»^{42}$

\section{De la résistance à l'émigration : la participation au complot de Lyon.}

Les Saint-Vincent peuvent être considérés comme des contrerévolutionnaires au sens strict du terme : ils protestent avec les parlementaires, ils refusent la mort des privilèges ${ }^{43}$. Le paradoxe réside dans le fait qu'ils demeurent hostiles à la monarchie absolue. La cour, les ministres relèvent des catégories de l'intrigue et du despotisme, toujours suspects de « jésuitisme ». Ils sont plus royalistes que le roi, au nom de la critique du pouvoir absolu du roi qu'ils appellent despotisme ministériel. Pour Robert le roi n'est roi qu'en son Parlement, un peu sur le modèle anglais $\mathrm{du}$ « King-in-Parliament $»^{44}$. À aucun moment il ne parle du sacre, du roi thaumaturge ou du caractère sacré de la monarchie. Et pourtant la famille entière va participer à l'un des premiers complots pour sauver Louis XVI. Robert décrit avec minutie toutes les avanies symboliques subies de la part de ses paysans ; mais il demeure vague et allusif sur les motivations et les circonstances qui l'entraînent dans l'aventure du complot de Lyon.

Robert de Saint-Vincent, partisan de l'ordre et de la tradition, ne s'est pas contenté de protestation secrète. Dans sa correspondance avec l'abbé Clément, il demandait déjà en mai 1789 la mise en action du réseau janséniste européen ${ }^{45}$. Il fallait prévenir la destruction des parlements et de la religion car Robert se félicite dans ses Mémoires d'avoir été immédiatement convaincu « que la Révolution aurait la plus funeste issue ${ }^{46}$. Il s'est donc engagé fort tôt dans l'action. Ce fut une belle surprise de découvrir dans ses Mémoires qu'il fut impliqué dans l'un des premiers complots visant à faire évader Louis XVI. Conçu par Mirabeau, organisé par le club du

(42) II, p. 212.

(43) Ils sont hostiles à toutes les nouveautés depuis mai 1789, et même septembre 1788 , mais la restauration qu'ils souhaitent n'est pas celle de la monarchie absolue qui semble ne jamais avoir existé pour eux, sinon comme abus ; il faut restaurer la monarchie des juges. Michel FIGEAC, op. cit. François LEBRUN et Roger DUPUY (dir.), Les résistances à la Révolution, Actes du Colloque de Rennes (17-21 septembre 1985), Paris, Éd. Imago, 1987 ; Jacques GODECHOT, La Contre-Révolution (1789-1804), Paris, PUF, 1984 ; Jean-Clément MARTIN, «Introduction », dans Jean-Clément MARTIN (dir.), Dictionnaire de la Contre-Révolution, Paris, Perrin, 2011, p. 7-28. $127-150$.

(44) Bernard CotTreT, «Le Roi, les Lords et les Communes », Annales ESC, 1986, 41, p.

(45) Lettre 13, 17 mai 1789.

(46) II, p. 206. 
Salon français ${ }^{47}$ en liaison avec les émigrés de Turin, le plan d'évasion prévoyait à l'occasion d'une chasse à Fontainebleau la fuite du roi vers Lyon où il aurait été rejoint par une armée piémontaise, des troupes suisses et espagnoles. Présenté à Mme Élisabeth en juillet 1790, ce plan avait été rejeté par le roi qui avait ordonné au comte d'Artois d'y renoncer. Celui-ci avait pourtant réussi à arracher à son frère la promesse d'un départ pour le 15 décembre $1790^{48}$.

C'est à ce projet qui fut une fois de plus repoussé par le roi et dénoncé publiquement, que Robert de Saint-Vincent fut associé par l'entremise de Pierre-Jacques Le Maître ${ }^{49}$ avec qui il était en correspondance. Janséniste, émule de La Chalotais, opposant à la réforme de Maupeou, autant de titres propres à le rendre sympathique et fiable aux yeux de Robert de Saint-Vincent :

« ce digne homme en qui j'ai toujours trouvé beaucoup d'esprit, de finesse et un très grand courage, était alors comme le conseil universel de tous ceux qui étaient ennemis de la révolution. Depuis le jour de la sortie de M. le comte d'Artois, M. Le Maître n'avait pas cessé de diriger son conseil, et par ses relations très sûres et très bien choisies dans toutes les cours d'Europe, il était en état de donner des conseils très utiles suivant le moment et les circonstances. Ce fut M. Le Maître qui m'instruisit de Paris qu'il y avait un projet d'entrée des princes de Turin, où ils étaient, par Lyon dans l'intérieur du royaume pour opérer une contre-révolution utile au roi contre les entreprises de l'Assemblée nationale [...]. J'étais instruit dans mon château de Fessard qu'il se préparait un mouvement intéressant de la part de M. le comte d'Artois et de M. le prince de Condé qui avaient un puissant parti du côté méridional de France, qu'il entrait dans ce plan que plusieurs magistrats se rendissent des différentes parties du royaume près les princes pour y composer un tribunal imposant qui établît les maximes du royaume dans le même moment où le succès de l'armée des princes

(47) Jacques de SAINT-VICTOR, « L'action parlementaire et les clubs contre- révolutionnaires », dans Jean TULARD (dir.), La Contre-Révolution : origines, histoire, postérité, Paris, CNRS éditions, 2013, p. 35-54.

(48) Louis XVI envoie le baron de Vioménil à Turin début août pour rappeler que les princes ne peuvent agir sans son autorisation. Le comte d'Artois demande néanmoins à son frère s'il accepterait de quitter Paris en octobre. La réponse, négative, arrive à Turin le 2 octobre. Puis Louis XVI, pressé par le chanoine de Rully, accepte de partir avant le 15 décembre, Ghislain de DIESBACH, Histoire de l'Émigration (1789-1814), Paris, Perrin, 1998, p. 104-109.

(49) Pierre Jacques Le Maître (1742-1795) fut l'un des agents actifs du comte d'Antraigues pendant la Révolution. André DOYON, Un agent royaliste pendant la révolution : Pierre-Jacques Le Maître, Paris, Société des études robespierristes, 1969. II, p. 219 ; Jacques GODECHOT, « Les réseaux contre-révolutionnaires » dans Jean TULARD (dir.), La Contre-Révolution, ... op. cit., p. 168-184. 
assurerait la couronne sur la tête du roi. Je fus averti dès le mois d'octobre de me tenir prêt à faire ce voyage $»^{50}$.

Louis XVI et Marie-Antoinette ne voulaient pas devoir la restauration de leur pouvoir à la noblesse dirigée par les princes, c'est pourquoi ils refusèrent de se prêter aux différents projets d'évasion plus ou moins bien élaborés par l'entourage de ces derniers. Robert de Saint-Vincent aurait bien voulu que le roi soit également redevable aux magistrats garants des lois fondamentales du royaume. Il associe d'ailleurs à cette glorieuse entreprise son fils Pierre-Antoine ${ }^{51}$ et son gendre ${ }^{52}$ « tous deux magistrats du Parlement que je ne voulais pas trop laisser en arrière afin qu'ils prissent part aux démarches courageuses que le Parlement devait faire en cette occasion $\gg^{53}$. Résolument du côté des princes et de l'intervention extérieure, il confie au curé de Fessard en novembre-décembre 1790 que « s'il était nécessaire pour faire cesser la Révolution que les armées étrangères entrassent en France, je ne doutais pas que Dieu qui protège la France ne réduisît en poussière tous ces projets qui n'étaient que des verges envoyées contre la France pour la punir de son incrédulité et de son infidélité en tout genre $»^{54}$. Le projet des princes semble donner enfin au Parlement le rôle législatif qu'il ambitionne depuis longtemps : «établir les maximes du royaume ». Robert assume sans hésitation la « contre-révolution », pour le roi, contre l'Assemblée nationale.

Le voilà donc, en bon conspirateur, prêt à partir dès le signal donné. Pourtant il affirme en 1797 n'avoir eu « aucune idée d'émigration ${ }^{55}$, en particulier à cause de son épouse ${ }^{56}$. Il oublie alors combien il avait été confiant dans le succès du complot ! Il croyait effectivement à la réussite rapide de l'opération à laquelle il s'apprêtait à participer ${ }^{57}$. Mais l'affaire est annulée, puis dénoncée ${ }^{58}$. Impliqués et paniqués, craignant que leur père ne soit déjà en route pour Lyon, son fils et son gendre sont poussés à partir par Le Maître. Ils arrivent à Fessard le 18 décembre munis de passeports pour Genève. Robert ne songe pas à « quitter sa patrie » mais il

(50) II, p. 220.

(51) Pierre Antoine Robert de Saint-Vincent (1756-1826).

(52) Jean-Michel Le Chanteur (1764-1835).

(53) II, p. 220.

(54) Ibidem.

(55) II, p. 211.

(56) Ibidem.

(57) II, p. 220.

(58) Ghislain de DIESBACH, op. cit., p. 110-111. «L'affaire de Lyon était totalement manquée, soit par les entraves qu'y avait mis la reine de France, soit par d'autres obstacles dont je n'ai pas assez connu le détail pour en pouvoir parler ici. », II, p. 220. 
y est contraint. Il ne souhaite pas laisser son épouse et son dernier fils seuls face à une éventuelle répression. L'énergie de Mme de Saint-Vincent, prête à le suivre, décide de leur départ. Voici Robert de Saint-Vincent « conduit à m'expatrier sans presque m'en apercevoir ${ }^{59}$ nous dit-il. Certes, mais ce n'est pas par hasard que ses fils ont sollicité et obtenu des passeports pour Genève. Partis le 20 décembre dans le plus grand secret, les apprentis conspirateurs arrivent à Genève le 28 décembre.

\section{Un destin politique en émigration ?}

$\mathrm{Au}$ travers du récit de ses misères et pérégrinations (voir carte) Robert de Saint-Vincent donne des éléments qui permettent de reconstituer les débats politiques et les projets de ces premières années de l'émigration autour des princes, et alors que chacun pense que les puissances européennes vont anéantir la France révoltée. Robert a rallié sans état d'âme le parti des princes ; il se défie de la cour et des ministres. Il méprise Louis XVI et déteste la reine. Il continue de défendre la cause des magistrats qui pour lui se confond avec celle de la monarchie. C'est Condé qui le secoue lors de son passage à Genève et lui ordonne de rejoindre le comte d'Artois à Mayence ${ }^{60}$; Robert est enthousiaste et il se souvient douloureusement de ces faux espoirs : «les princes prenaient le chemin de la gloire pour nous reconduire avec éclat dans notre patrie ${ }^{61}$. Mais la gloire et l'éclat ne sont pas les caractéristiques de la vie politique en émigration. Dans ce petit milieu d'exilés se retrouvent toutes les frustrations et contradictions de l'Ancien Régime agonisant. La rencontre tant attendue avec le comte d'Artois se révèle bien amère : les magistrats n'ont pas été conviés au grand dîner donné en l'honneur du prince. Robert de Saint-Vincent proteste contre cet oubli « qui semblait tenir du mépris pour la robe $»^{62}$. Visiblement Artois a tiré les conséquences de cet épisode : il ménage désormais la susceptibilité du magistrat en le mettant «à la tête de son conseil ». Mais il lui impose la présence de Calonne, que Robert déteste, quitte à « arbitrer » leurs «petits débats ${ }^{63}$. Quelle place réelle les princes entendaient-ils réserver au Parlement dans une France renouant avec la monarchie ? Cela reste bien flou, mais le témoignage de Robert de Saint-Vincent demeure un indice précieux.

(59) II, p. 211.

(60) II, p. 241.

(61) Ibidem.

(62) II, p. 257. Finalement, les quatre plus anciens magistrats présents sont invités, p. 259.

(63) II, p. 266. 
La question de la régence est très vite abordée, dès que Louis XVI, après le 10 août 1792, se trouve enfermé au Temple. Le comte de Provence est contraint de respecter, au moins en apparence, les consignes de Louis XVI, qui sont transmises par Breteuil. Mais Robert continue la fronde parlementaire : il faut profiter de l'occasion pour donner la régence au frère du roi. C'est avec Barmont ${ }^{64}$ qu'il défend en conseil l'idée de la régence du royaume « qui ne pouvait rester sans gouvernement ». Monsieur repousse une régence qu'il n'a pas les moyens de soutenir. Robert avoue qu'il fut alors « très fâché qu'on ne profitât pas d'un moment » qui lui «paraissait unique dans l'histoire de la monarchie pour assurer sur la tête du premier prince du sang un droit qui pourrait lui être contesté par d'autres qui y prétendraient ${ }^{65} »$. Il décide de repartir à Leuze, trop chagriné de voir manquer « cette occasion unique ». En réalité, il a mesuré la divergence entre le couple royal et les princes et, s'il penche incontestablement du côté de Monsieur et ne démord pas de son aversion pour la reine, il veut s'éloigner « afin, dit-il, de ne me point compromettre par aucun propos qui pût offenser personne. » Il faudra presque un an avant qu'il soit de nouveau appelé.

Dès l'entrée en guerre, alors que tous attendent une victoire imminente, il est convoqué à Coblence en avril 1792 par les princes. Ceux-ci si l'on en croit Robert « imaginèrent que les magistrats pourraient être de quelque ressource $»^{66}$. Monsieur et le comte d'Artois lui auraient déclaré « qu'ils n'étaient pas fâchés d'avoir auprès d'eux une réunion de magistrats dont (il serait) l'ancien et qui pourrait en attirer d'autres, soit de Paris, soit des différentes provinces du royaume $»^{67}$. Cette version va à l'encontre d'une tradition historiographique selon laquelle les princes ne souhaitaient pas voir se reconstituer une cour qui avait par son opposition systématique contribué à déstabiliser le trône. D'après Henri Dugon, ce furent les magistrats émigrés, notamment Robert et Barmont, soucieux de contribuer au maintien des institutions, qui prirent l'initiative et obtinrent du duc de Piney qu'il s'entremette auprès

(64) Charles-François Perrotin de Barmont (1759-an IV). Conseiller-clerc au Parlement (1781), député du clergé de Paris hors les murs aux États généraux, secrétaire de la Constituante le 16 août 1789. Bientôt suspect, brièvement arrêté été-automne 1790 pour avoir caché M. de Bonne-Savardin évadé de l'Abbaye, il émigre en Allemagne.

(65) II, p. 273-274. Selon Robert, Calonne aurait réfuté l'idée d'une régence dans ce conseil. Ernest Daudet affirme au contraire que Calonne la suggéra, Ernest DAUDET, Histoire de l'émigration pendant la révolution française, Paris, Vve Ch. Poussielgue, 1904, vol. I, p. 75.

(66) II, p. 276.

(67) II, p. 281. 
des princes ${ }^{68}$. Illusion ou réalité ? Le récit de Robert le présente plutôt dans une assez grande proximité avec ceux-ci. Les magistrats sont finalement réunis à Mannheim. Si les Mémoires de Robert de Saint-Vincent offrent une version différente de celle laissée par le Président de Vezet ${ }^{69}$ (chacun se jugeant fondé à être à la tête de la cour), tous deux relatent les mêmes navrantes et dérisoires querelles de préséance. Envoyé à Luxembourg pour en réunir le plus grand nombre possible, Robert, qui semble plus enthousiaste que le Président de Vezet, y retrouve M. Lefebre d'Amécourt ${ }^{70}$. Mais celui-ci n'a pas «la même boussole » et ne suit pas « les mêmes ordres ». Proche du baron de Breteuil, d'Amécourt lui apprend qu'un autre projet est en cours, la réunion d'un corps de magistrature à Metz ou Verdun, et que tous les magistrats devront rentrer en France dès qu'ils en auront reçu l'ordre. Le plan semble insensé et n'a pas laissé de traces. Robert de Saint-Vincent réaffirme à l'occasion sa défiance à l'égard de Breteuil et son engagement auprès des princes ${ }^{71}$. Ce qui ne l'empêche pas de s'insurger lorsqu'il apprend que la présidence de cette future cour devrait être donnée à $\mathrm{M}$. de Barentin ${ }^{72}$. Il multiplie fébrilement les mémoires sur les principes du Parlement. Valmy met fin à ces chimères :

« Nous fûmes tous confondus lorsque, au moment où nous ne pensions qu'à une victoire complète, nous apprîmes la retraite de l'armée des princes et le congé par eux donné à toute la noblesse qui les avait accompagnés. Nous eûmes le sang gelé dans nos veines quand nous apprîmes que, loin d'avancer vers la France, nous étions invités à nous retirer chacun dans le lieu de nos retraites respectives $»$.

Tous les espoirs de Robert s'effondrent, il abandonne définitivement toute velléité d'action politique.

(68) Henri Dugon, Au service du roi en exil : épisodes de la Contre-Révolution d'après le journal et la correspondance du président de Vezet (1791-1804), Mâcon, impr. Protat frères, 1968, p. 23 et $s q$. D'après Henri Forneron, ce fut Barentin qui, pour se faire pardonner la lâcheté de son gendre Dambray, se chargea de réunir une cour plénière à Mannheim. Henri FORNERON, Histoire générale des émigrés pendant la révolution française, Paris, Plon, Nourrit, 1884, vol. 1, p. 327. Le trop petit nombre de magistrats (50) les amena à conclure qu'ils ne pouvaient « se donner comme les interprètes légitimes de la magistrature », Alexandre ESTIGNARD, Le parlement de Franche-Comté de son installation à Besançon à sa suppression, 1674-1790, Paris, A. Picard, 1892, vol. 2, p. 376.

(69) Joseph Luc Jean-Baptiste Hyppolite Mareschal de Vezet (ou de Mareschal-Vezet), comte (1743-1816). Président du parlement de Besançon.

(70) Adrien Lefebvre d'Amécourt (1720- ?). Conseiller à la $3^{\mathrm{e}}$ chambre des Enquêtes en 1740, émigré en 1792.

(71) II, p. 303.

(72) Charles Louis François de Paule de Barentin (1738-1819), président de la Cour des aides en 1775. Louis XVI le nomme garde des Sceaux en 1788, il mène l'opposition à Necker et doit démissionner après le 14 juillet. 
Monsieur, régent du royaume, aurait-il reconstitué les cours souveraines et restauré les parlements et la noblesse de robe ? Robert de Saint-Vincent en est persuadé, mais est-il un bon observateur ? L'essentiel de son activité consiste à rassembler un Parlement de l'exil et Dieu sait que l'affaire n'est pas simple entre partisans du roi et partisans des princes, entre anciens et modernes, entre Paris et les provinces, au milieu des conflits de préséance et des rivalités entre les corps. Ce que nous apprenons par contre, c'est que les princes ont besoin des magistrats pour exercer la justice, et en l'occurrence la justice politique, lors de leur retour triomphal dans cette France qui est supposée attendre la délivrance austro-prussienne et royaliste. À cet effet, ils ont mandaté Robert, qui a accepté le rôle sans hésitation. Il s'agit de « faire quitter le service militaire à ceux des magistrats qui depuis l'émigration s'étaient laissés aller à leur zèle pour servir militairement le roi de France et sa famille auxquels ils ne pouvaient plus rendre aucun service en qualité de magistrat $\gg^{73}$. Cette confusion désole Robert qui croit en la dignité de la robe face à l'épée. Les magistrats du parlement de Rennes qui ne veulent pas abandonner l'état militaire trahissent leur monde et n'accomplissent pas le service véritable et honorable de la monarchie. À cette raison fondamentale, la dignité et l'utilité de la fonction, s'en ajoute une beaucoup plus pragmatique :

«La question avait été plus d'une fois discutée en présence des princes et ailleurs, et il avait été décidé par les principes de la raison et de la justice, qu'il ne convenait nullement que les mêmes personnes [...] poursuivissent l'épée à la main les révolutionnaires et les rebelles contre le roi, en se réservant le droit de les faire ensuite exécuter sur un échafaud si le sort des armes rendait les rebelles leurs prisonniers ${ }^{74}$.

Pour qui en aurait douté, les émigrés qui, en 1792, pensaient reconquérir le royaume n'arrivaient pas dans un esprit de pardon et d'oubli, mais avec les armes de la justice. Quand on songe que pour Robert de Saint-Vincent, la rébellion avait commencé avec le doublement du Tiers, c'était faire une bien large place à l'échafaud...

La lutte commune contre la rébellion française ne signifie pas que les vieilles querelles soient éteintes : les jésuites continuent de persécuter les jansénistes. Robert de Saint-Vincent et son épouse désormais infirme sont menacés par le prince de Liège qui leur demande de quitter au 
plus vite son territoire. Ils ont été dénoncés comme mauvais catholiques, probablement par le « jésuite » Feller ${ }^{75}$ confie Robert de Saint-Vincent ; mais à cette occasion, le comte de Provence maintient sa protection et écrit personnellement au prince : «J'ai appris avec peine, Monsieur et cousin, le désagrément qu'avait éprouvé M. Robert de Saint-Vincent, conseiller au Parlement de Paris. Depuis que je le connais, j'ai entendu parler de ce magistrat comme un des plus respectables, et depuis ma sortie de France, j'ai mieux apprécié ses vertus dont le respect et la pratique de la religion fait la base... Louis Stanislas Xavier $\gg^{76}$. On imagine la satisfaction de notre magistrat qui transcrit fidèlement cette attestation de fidélité et de catholicisme. Mais les offensives anti-jansénistes se poursuivent néanmoins. Lors de son arrivée à Utrecht, Robert se trouve sans ressources ; il est alors secouru par ses amis de l'Église d'Utrecht. Ceux-ci éprouvent sa modestie en lui donnant d'abord fort peu des mains d'une femme de rien, puis ayant constaté son humilité, lui offrent de quoi entretenir sa famille et, précise-t-il, de «payer le loyer des différents appartements que nous fûmes dans le cas d'occuper $\gg^{77}$. Est-ce cette générosité qui exaspère les autres émigrés ? M. de Barentin, M. d'Aligre lui font savoir que le clergé émigré est particulièrement scandalisé par le fait qu'il fréquente une Église qu'ils considèrent comme schismatique et janséniste. Robert s'indigne :

« Je crus devoir à la vérité, à la justice et à l’Église de Hollande de prendre la défense de l'Église de Hollande en me déclarant hautement pour la canonicité de l'élection de ses prélats [...]. On prétendait à La Haye, où résidaient quatorze évêques, me rendre responsable par mon exemple de la conduite de tous ceux des Français qui continueraient à fréquenter les églises de ces vénérables évêques $»^{78}$.

Cette fois Robert n'a pas le soutien des princes :

«M. de Barentin, qui était poussé par les évêques émigrés et qui croyait se faire un grand appui dans ce vœu réuni de l'épiscopat français émigré, poussa la pointe pour me réduire soit par le pouvoir de M. le comte d'Artois qui était alors peu éloigné de La Haye, soit par l'autorité de Monsieur alors régent, par lequel il me fit écrire une lettre capable de m'intimider si j'avais

(75) François-Xavier de Feller (1735-1832), ex-jésuite et polémiste contre les Lumières et la Révolution. II, p. 375-376.

(76) II, p. 377-378.

(77) II, p. 389-390.

(78) II, p. 391. L'Eglise catholique de Hollande a été le refuge de tous les dissidents, surtout depuis sa rupture avec Rome en 1724. 
pu l'être en pareille matière. Je répondis à Monsieur avec tout le respect que je lui devais et avec la fermeté dont Monsieur me savait capable. Je ne fus point intimidé par les espérances de faveur, ni par les craintes de disgrâces et je montrai à $\mathrm{M}$. de Barentin qu'il s'adressait mal en voulant soutenir par l'autorité une affaire dont il n'avait pas la première idée juste ${ }^{79}$.

Le témoignage de Robert de Saint-Vincent est conforté par celui de l'abbé Grégoire, qui bien plus tard rapporte la même aventure ; Barentin tente d'imposer à Robert de rompre avec l'Église d'Utrecht : « il le menaçait du stadhouder s'il continuait d'aller à l'église archiépiscopale » ${ }^{80}$... Les armées républicaines qui envahissent le pays mettent provisoirement fin à la vieille querelle.

\section{L'effondrement d'un monde}

Les deux Mémoires, que la chronologie croisée de leur rédaction rend indissociables ${ }^{81}$, conjuguent la volonté d'apporter aux générations futures un témoignage et le besoin d'épanchement d'un vieil homme pour qui drame personnel et naufrage collectifs se confondent. Racontée pratiquement à chaud, ce qui est exceptionnel, la plupart des mémoires d'émigration connus ayant été écrits sous la Restauration ou dans la seconde partie du XIX ${ }^{\mathrm{e}}$ siècle, la tragédie personnelle de Robert de Saint-Vincent commence avec celle du royaume : "Je ne veux en parler ici que relativement à Mme de Saint-Vincent à qui il est très vrai de dire que la Révolution a donné la mort. On peut juger combien il me sera pénible de me rappeler toutes les anecdotes qui lui sont personnelles dans cette douloureuse catastrophe qui a inondé la France de sang et de carnages $»^{82}$.

Mais, comment dire ce « moi haïssable » ? Et comment résister à cette « propulsion de tout son être, vers ce qui peut lui apparaître comme un repère sûr susceptible de lui rendre, sinon la belle solidité de naguère,

(79) II, p. 392.

(80) Henri GréGOIRE, Mémoires, 2 vol., Paris, J.Youet, 1840, tome 2, p. 185.

(81) Le Mémoire de famille a été commencé en 1795 à Wandsbeck. Sa rédaction a été interrompue (p. 466) par celle du second Mémoire pour sa femme, entreprise, à la suite de son décès, de janvier 1797 à fin février 1799. Robert n'a repris la rédaction de son premier mémoire qu'en 1798 pour le laisser (hélas !) inachevé à sa mort en 1799. La dernière mention de date est de mai 1799.

(82) II, p. 172. Ainsi que le souligne Anne Coudreuse, "c'est donc finalement l'événement révolutionnaire et la situation politique qui génèrent l'écriture de l'intime, et non pas le thème macabre », Anne CoudREUSE, «L'intime et le politique dans les Mémoires du marquis de La Maisonfort », Anne Coudreuse, Françoise Simonet-Tenant (dir.), Pour une histoire de l'intime et de ses variations, Paris, Éditions L'Harmattan, 2009, p. 99. 
du moins le souvenir qu'il peut en conserver ${ }^{83}$ ? Robert se dédouane tout d'abord de la vanité qui est « presque toujours l'objet principal de ces sortes de mémoires » en imputant l'initiative de son entreprise d'écriture à son fils aîné. C'est en effet celui-ci qui le " presse », nous dit-il, de « confier au papier et peut-être à la postérité ce qui me reste de notion sur mon nom et ma famille» ${ }^{84}$. Moyen privilégié d'assurer la survie de la caste, le tableau récapitulatif des origines de la famille permet de parler du monde et de soi dans le monde, de fixer pour la postérité la fortune enfuie. Si l'homme affleure, dans le tri opéré, c'est le magistrat, représentant statutaire d'un corps, qui est mis en avant. Autre contournement du «moi », la volonté de « graver dans le cœur de sa postérité le respect et la vénération dus à la mémoire de cette digne et respectable mère de famille qui doit servir de modèle à toutes celles qui porteront son nom ${ }^{85}$ préside formellement à la rédaction du second mémoire. Pourtant, il avoue qu'il écrit surtout pour lui, pour fuir la peine du temps présent et, en se replongeant dans le passé, échapper à la tristesse et au vide, au naufrage d'un monde dont le rappel doit faire sentir tout ce qu'il a perdu : « Je ne puis éprouver de consolation que dans le récit simple que je veux faire pour moi et pour mes enfants à qui j'en suis bien aise d'en conserver la mémoire, de la douceur et du bonheur dont j'ai joui avec elle pendant plus de quarante-deux ans de ma vie $»^{86}$. Contradiction d'un homme qui dit forcer sa nature en parlant de lui, et qui trouve dans l'écriture l'indispensable exutoire à sa douleur. Difficulté à concilier l'humilité chrétienne à laquelle il aspire avec le troublant et périlleux exercice de se dire. Comment fuir la nécessaire vanité ? Robert de Saint-Vincent, en bon janséniste, trouve une dernière échappatoire en transformant ses récits en actions de grâce : quel « prétexte plus honnête et de plus légitime que de faire le plus brièvement qu'il me sera possible le récit des miséricordes de Dieu sur ma personne ${ }^{87}$ ?

Si l'écriture lui offre un refuge, Robert se laisse pourtant aller à quelques questions désabusées : «À quoi bon parler de noblesse et de titres à ses descendants quand ce nom, ces titres et ces avantages les ont rendus odieux à la génération présente ? À quoi bon leur apprendre que leurs pères

(83) François JACOB, Henri ROSSI, Mémorialistes de l'exil : émigrer, écrire, survivre, Paris, Éditions L'Harmattan, 2003, p. 10 ; Karine RANCE, « Mémoires de nobles émigrés dans les pays germaniques pendant la Révolution française », thèse de doctorat sous la direction d'Étienne FRANÇOIS, Université Paris I-Panthéon-Sorbonne, 2001 ; Philippe BoURDIN, « Mémoires d'ex-, mémoires d'exil : l'émigrante noblesse auvergnate », AHRF, 343, janvier-mars 2006, p. 3-27.

(84) I, p. 3.

(85) II, p. 1.

(86) Ibidem.

(87) I, P. 133 
et leurs aïeux ont été dans l'opulence pour ne leur laisser que le souvenir de tout ce qu'ils ont perdu en ce monde et leur causer des regrets cuisants et amers s'ils sont destinés à vivre dans l'indigence que la privation de toute notre fortune nous donne aujourd'hui ? ${ }^{88}$ Moyen de surmonter la réalité ou de la fuir ? Entreprise de survie ou recherche du temps perdu ? La mort de son épouse fait basculer le récit. Il s'agit alors à l'évidence d'une fuite, fuite de la douleur, de la solitude, du chaos du temps présent. Il parcourt les grands événements de sa vie comme autant de stations du chemin de croix qui ont amené sa femme au tombeau. Hanté par la crainte d'avoir ajouté aux peines de sa femme, il passe en revue ses erreurs, les coups qui ont précipité son malheur jusqu'à ce que la mort lui arrache « la moitié de moi-même, celle que j'aimais le plus tendrement et qui a fait mon bonheur pendant plus de quarante-deux ans de ma vie $»^{89}$.

Si la peine préside à l'écriture, le retour dans le passé permet à Robert de Saint-Vincent de revivre les bons moments, se rappelant leurs crises de rire, leurs dialogues, les gestes tendres. Autant de scènes qui ouvrent les portes de leur intimité et donnent à voir le fonctionnement de leur couple ${ }^{90}$, des premières paroles échangées aux derniers regards d'amour pathétiques. La fin du récit, cette mise en scène de deux vieux amants qui se quittent, est poignante. À ce stade du texte, l'homme est dépouillé de tout artifice. Il est désormais « tout à fait isolé dans le monde » ${ }^{91}$.

Au-delà de l'intime, dans la ligne des grands récits d'émigration, Robert nous emmène sur les routes d'Europe. Il s'étonne, au milieu de son chagrin, des caprices qui ont mené leur vie au fin fond du Holstein, il ne revient « pas encore de l'idée qu'une femme née à Orléans [...] soit venue terminer sa carrière au village de Wandsbeck dans le Holstein ! ${ }^{92}$ En Suisse où il indispose un calviniste genevois par ses questions sur la Réforme et n'a de cesse de voir les originaux du concile de Bâle ${ }^{93}$, en Allemagne où la vie est encore douce aux émigrés, au Luxembourg, en Belgique où il apprend la mort du roi ${ }^{94}$, à Utrecht, il nous plonge dans le petit monde des émigrés, nous faisant revivre les réceptions princières des premiers temps, les intrigues contre-révolutionnaires, le premier siège de Maastricht ${ }^{95}$,

(88) I, P. 1.

(89) II, p. 404.

(90) II, p. 69.

(91) II, p. 455.

(92) II, p. 2.

(93) II, p. 239 et 242.

(94) II, p. 328-332.

(95) II, p. 332-361. 
l'apparition de la gêne financière, de l'hostilité des populations ${ }^{96}$, jusqu'à ce château de Wandsbeck où il va vivre son plus grand drame.

$\mathrm{Au}$ final qui était Robert de Saint-Vincent? Les ego-documents analysés dans cet article permettent de retrouver la cohérence du personnage, de tisser le lien entre le bouillant opposant au despotisme ministériel, le farouche janséniste qui défend la tolérance civile à l'égard des protestants, et l'aristocrate qui s'accroche aux privilèges. Pour lui la Révolution est vraiment un choc. En quelques mois, tout devient ennemi ! Robert de SaintVincent a traversé le siècle des Lumières avec la nostalgie du classicisme, d'un style français, gallican, reposant sur la tradition juridique, l'histoire et l'érudition. Une histoire largement mythique, certes, et une érudition partielle, faite de références répétées, mais qui lui fournissent des arguments solides contre toutes les nouveautés. Que ce passé puisse se transformer en alibi pour la Révolution, que ses amis rallient le Tiers et abandonnent les privilèges, voilà ce qu'il ne peut admettre. L'explication par le complot prend alors une ampleur déconcertante : tout est jésuite, les philosophes, les académiciens, les protestants, et... les jansénistes. L'hydre ignatienne malveillante recouvre le monde entier ! Robert est plus systématique encore que l'abbé Barruel dans cette vision du monde.

Robert de Saint-Vincent a cru en la monarchie de justice. Il n'a pas entériné ce que les historiens appellent la « révolution de 1661 ». Il interprète comme pur despotisme ministériel toute volonté absolutiste. Il défend la monarchie des juges contre Louis XV, Louis XVI et la Révolution. Lorsqu'il entre dans l'action aux côtés des princes, et toujours contre la cour, il est prêt à exercer une sévère répression contre la rébellion. Valmy met fin à cette terrible espérance ; pour Robert s'ouvre le temps du désespoir. Dieu est derrière cet immense cauchemar et Robert attend la fin. Il accorde à Robespierre une place particulière dans le musée des horreurs de la Révolution et a parfaitement assimilé de son lointain exil le langage thermidorien : pourtant à aucun moment « le tyran », « le monstre » Robespierre n'est assimilé aux jésuites. Pour Robert décidément tout est fini. 


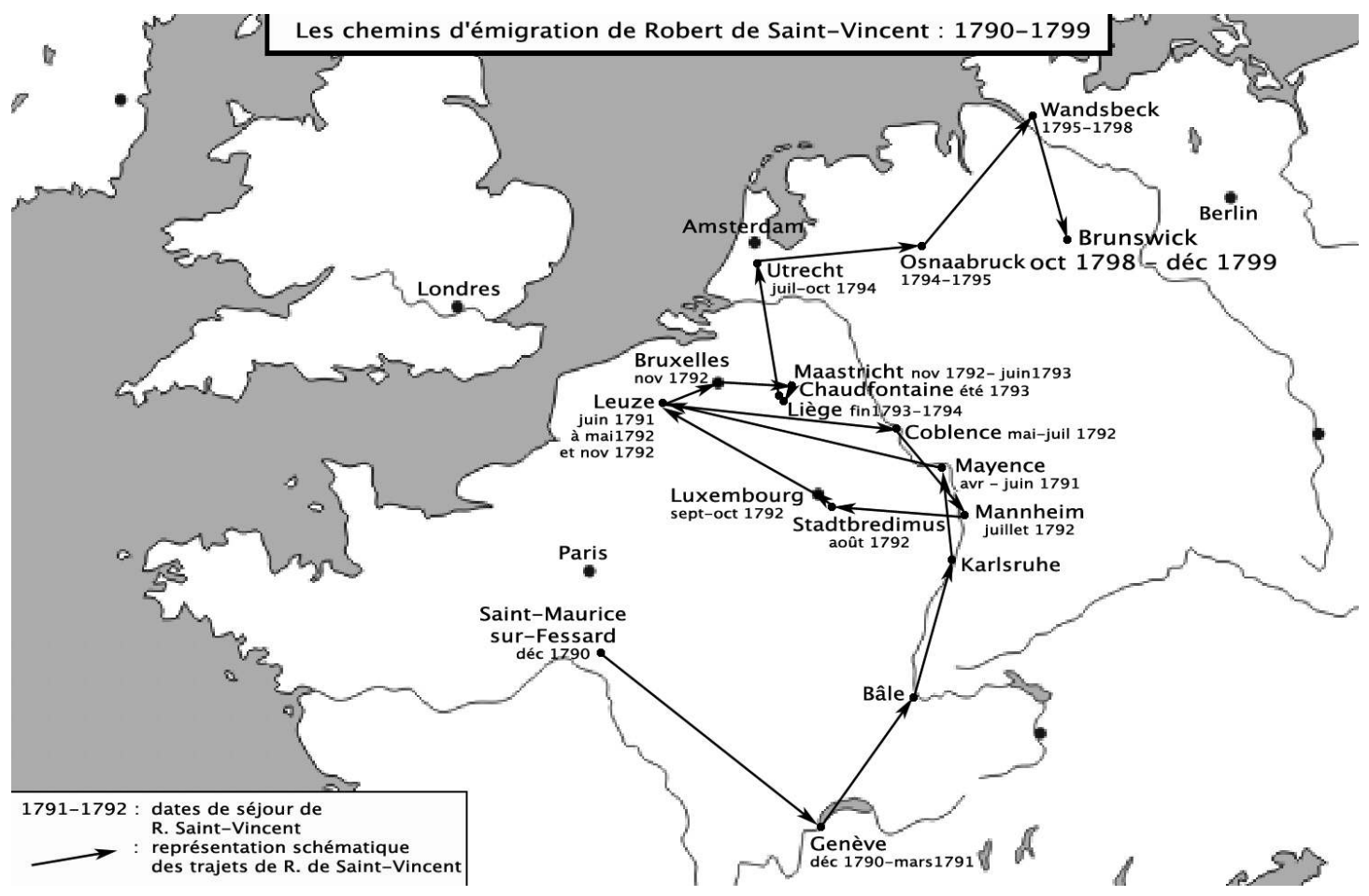

Figure 1.

Valérie GUITTIENNE-MÜRGER

Bibliothécaire Société de Port-Royal CHISCO, Paris Ouest-Nanterre-La Défense valeriemurger@voila.fr

Monique COTTRET CHISCO, Paris Ouest-Nanterre-La Défense monique.cottret@u-paris10.fr 